Trauma Berufskrankh 2017 · 19 (Suppl 2):S192-S197 DOI 10.1007/s10039-017-0246-x

Online publiziert: 6. März 2017

๑) Springer Medizin Verlag Berlin 2017

CrossMark
J. Hack · B. Bücking · D. Eschbach · S. Ruchholtz

Zentrum für Orthopädie und Unfallchirurgie, Universitätsklinikum Gießen und Marburg GmbH, Standort Marburg, Marburg, Deutschland

\section{Hüftendoprothetik nach Trauma}

\section{Was sind die „Big Points"?}

Schenkelhalsfrakturen sind typische Frakturen des alten Menschen, deren Häufigkeit in Zukunft durch den zunehmenden Anteil älterer Menschen an der Bevölkerung weiter ansteigen wird [22]. Bei jüngeren Patienten treten Schenkelhalsfrakturen wesentlich seltener und in der Regel nur nach Rasanztraumata auf.

Aufgrund der Besonderheiten des bei Schenkelhalsfrakturen meist osteoporotischen Knochens werden spezielle Anforderungen an die operative Versorgung gestellt. Zusätzlich erfordern das häufig fortgeschrittene Alter der Patienten und die damit einhergehende Multimorbidität besondere Maßnahmen im perioperativen Management.

Im vorliegenden Beitrag sollen deshalb einerseits die verschiedenen operativen Therapieoptionen bei Schenkelhalsfrakturen und andererseits die Besonderheiten im perioperativen Management der Patienten erläutert werden.

\section{Epidemiologie}

Die Inzidenz proximaler Femurfrakturen liegt in den Industriestaaten zwischen $150 / 100.000$ bis über $250 / 100.000$ [19], wobei etwa die Hälfte davon auf Schenkelhalsfrakturen entfällt [15]. Mit steigendem Lebensalter nimmt die Inzidenz von Schenkelhalsfrakturen deutlich $\mathrm{zu}[11]$.

\section{Ätiologie und Risikofaktoren}

Schenkelhalsfrakturen entstehen größtenteils im Rahmen von Bagatelltraumata, z. B. durch Stolperstürze oder Stürze aus niedriger Höhe. Kausal ist meist die im Alter oft vorliegende Kombination aus Sturzneigung und Osteoporose.
Bei jüngeren Patienten mit guter Knochenqualität liegt in der Regel ein Rasanztrauma zugrunde, beispielsweise ein Sturz aus großer Höhe oder ein Verkehrsunfall.

\section{Klassifikation}

Schenkelhalsfrakturen sind definiert als intrakapsuläre Frakturen, die zwischen Femurkopf und Trochanterregion gelegen sind.

Die Einteilung des Frakturtyps anhand von Klassifikationen spielt eine wichtige Rolle bei der Therapieplanung. Schenkelhalsfrakturen werden üblicherweise eingeteilt anhand der Klassifikation nach Pauwels, die die Stabilität der Fraktur beschreibt, und der Klassifikation nach Garden, die den Dislokationsgrad veranschaulicht.

\section{Klassifikation nach Pauwels}

Die Klassifikation nach Pauwels unterteilt Schenkelhalsfrakturen anhand des Frakturverlaufs in 3 verschiedene Typen [31]:

- Typ I: $<30^{\circ}$,

- Typ II: $30-50^{\circ}$,

- Typ III: $>50^{\circ}$.

Der Winkel zwischen Frakturlinie und der Horizontalen wird ausgemessen und anhand dessen die Stabilität der Fraktur beurteilt. Mit zunehmendem Winkel verringert sich die Stabilität der Fraktur.

Allerdings spielt die Pauwels-Klassifikation heute nur noch eine untergeordnete Rolle, da inzwischen nahezu alle Schenkelhalsfrakturen, unabhängig von der Stabilität, operativ versorgt werden.

\section{Klassifikation nach Garden}

Die Klassifikation von Garden beschreibt den Grad der Dislokation von Schenkelhalsfrakturen [14]. Die Dislokation nimmt von Typ I zu Typ IV zu.

- Typ I beschreibt valgisch eingestauchte, undislozierte Frakturen.

- Typ II beschreibt komplette, jedoch undislozierte Frakturen.

- Bei Typ III handelt es sich um partiell dislozierte Frakturen, bei denen medialseitig noch Kontakt zwischen den Fragmenten besteht.

- Typ IV sind deutlich dislozierte Frakturen.

Die Klassifikation nach Garden spielt eine wichtige Rolle bei der Entscheidung zwischen gelenkerhaltender Versorgung vs. Gelenkersatz, da mit zunehmender Dislokation die Blutversorgung des Femurkopfes schlechter wird.

\section{Klinik}

Der Patient gibt Leistenschmerzen und/ oder Schmerzen in der Trochanterregion an. In der Regel kann das betroffene Bein nicht mehr belastet werden. Bei dislozierten Frakturen ist das Bein durch den Muskelzug mit Überwiegen der Außenrotatoren typischerweise verkürzt und außenrotiert.

\section{Diagnostik}

Meist reichen zur Diagnosesicherung und Therapieplanung eine Beckenübersichtsaufnahme und eine axiale Röntgenaufnahme der betroffenen Hüfte aus.

Selten ist eine Computertomographie (CT)-Untersuchung notwendig, z. B. wenn klinisch der Verdacht auf 


\begin{tabular}{|c|c|c|c|}
\hline & $\begin{array}{l}\text { Dynamische Hüft- } \\
\text { schraube }\end{array}$ & Duokopfprothese & Totalendoprothese \\
\hline Alter & Alle & $>80$ Jahre & $<80$ Jahre \\
\hline Frakturtyp & Garden I-II & Garden III-IV & Garden III-IV \\
\hline $\begin{array}{l}\text { Vorbestehende sympto- } \\
\text { matische Koxarthrose }\end{array}$ & Nein & Nein & $\mathrm{Ja}$ \\
\hline ASA-Klassifikation & Alle & ASA 3-5 & ASA 1-2 \\
\hline Gehstrecke & Alle & Eingeschränkt & Uneingeschränkt \\
\hline Kognitiver Status & Alle & Dement & Nicht dement \\
\hline
\end{tabular}

eine Schenkelhalsfraktur besteht und anhand der konventionellen Röntgenbilder aufgrund ausgeprägter degenerativer Veränderungen eine undislozierte Fraktur nicht sicher ausgeschlossen werden kann.

Wenn über einen längeren Zeitraum Schmerzen in der Hüfte ohne Frakturnachweis im Röntgen oder in der CT bestehen, kann bei Verdacht auf Insuffizienzfraktur, also eine Fraktur ohne Trauma, in Einzelfällen auch eine Magnetresonanztomographie (MRT)Untersuchung sinnvoll sein.

\section{Therapie}

Schenkelhalsfrakturen müssen in den allermeisten Fällen operativ versorgt werden. Lediglich bei stabilen, nicht dislozierten, valgisch eingestauchten medialen Schenkelhalsfrakturen (Garden Typ I) ist ein konservativer Therapieversuch denkbar. Aufgrund der hohen Rate an sekundären Dislokationen (bis $33 \%$ [6]) und der Notwendigkeit der Entlastung, die bei geriatrischen Patienten vermieden werden sollte, versorgen wir im eigenen Vorgehen jedoch auch diese Frakturen primär operativ.

Die Art der operativen Versorgung von Schenkelhalsfrakturen hängt von verschiedenen Faktoren wie Frakturmorphologie, Alter, Aktivitätsniveau und Komorbiditäten des Patienten sowie einer ggf. vorbestehenden Koxarthrose ab. Prinzipiell finden sowohl osteosynthetische, das Hüftgelenk erhaltende als auch endoprothetische, das Hüftgelenk ersetzende Verfahren Anwendung. Einen Überblick der Indikationen zu den verschiedenen operativen Versorgungs- möglichkeiten bei Schenkelhalsfraktur gibt • Tab. 1.

\section{Osteosynthetische Verfahren}

Die osteosynthetische Versorgung von Schenkelhalsfrakturen erfolgt üblicherweise mittels dynamischer Hüftschraube (DHS), seltener mit 3 kanülierten Schrauben. Das Prinzip der DHS beruht darauf, dass die Schenkelhalsschraube dynamisch in ihrer Hülse gleiten kann und die Fraktur bei Belastung durch Sinterung weiter impaktiert wird. Durch die hierbei auf den Frakturspalt ausgeübte Kompression soll die Frakturheilung gefördert werden.

Bei (biologisch) jungen Patienten (bis etwa 60 Jahre) ist eine osteosynthetische Versorgung prinzipiell bei jedem Frakturtyp indiziert. Von entscheidender Bedeutung ist hier ein früher Operationszeitpunkt, möglichst innerhalb von $6 \mathrm{~h}$ nach dem Unfall, da dadurch das Risiko des Auftretens einer Hüftkopfnekrose im Verlauf deutlich verringert wird [27].

Bei geriatrischen Patienten werden lediglich eingestauchte oder nicht dislozierte Schenkelhalsfrakturen (Typ I und II nach Garden) osteosynthetisch versorgt, um eine sekundäre Dislokation der Fraktur zu verhindern. Eine Osteosynthese ist bei älteren Patienten außerdem nur dann sinnvoll, wenn keine symptomatische Koxarthrose vorliegt.

Neben dem Vorteil des Gelenkerhalts beim jungen Patienten werden als weitere potenzielle Vorteile der Osteosynthese gegenüber der endoprothetischen Versorgung eine kürzere Narkosedauer, weniger Blutverlust und eine niedrigere Transfusionsrate angeführt $[29,30]$.
Potenzielle Nachteile liegen in der Nachbehandlung, da teilweise zunächst nur eine Teilbelastung freigegeben werden kann, in der technisch anspruchsvollen Implantatpositionierung und in möglichen Komplikationen wie Osteosyntheseversagen (insbesondere Cutting-out und Hüftkopfperforation mit konsekutiver Schädigung der Pfanne, - Abb. 1), Femurkopfnekrose, sekundärer Koxarthrose oder Pseudarthrose, besonders bei dislozierten Frakturen (Typ Garden III und IV). Bei dislozierten und auch undislozierten Schenkelhalsfrakturen ist die Revisionsrate nach osteosynthetischer Versorgung signifikant höher als nach endoprothetischer Versorgung [17, 29].

\section{Endoprothetische Verfahren}

Aufgrund der zuvor beschriebenen Komplikationen ist bei geriatrischen Patienten mit dislozierter Schenkelhalsfraktur (Typ Garden III und IV) ein endoprothetischer Gelenkersatz indiziert. Gjertsen et al. [17] konnten zeigen, dass Patienten, die eine osteosynthetische Versorgung bei dislozierter Schenkelhalsfraktur erhalten hatten, eine signifikant häufigere Reoperationsrate, signifikant mehr Schmerzen sowie eine schlechtere Lebensqualität aufwiesen als Patienten, die eine Duokopfprothese erhalten hatten.

\section{Totalendoprothese versus Duokopfprothese}

Bei der endoprothetischen Versorgung von Schenkelhalsfrakturen finden sowohl die Totalendoprothese (TEP) als auch die Hemiprothese Einsatz (• Tab. 1).

Als Hemiprothese wird üblicherweise die Duokopfprothese (bipolare Prothese) implantiert, bei der die "Artikulation“ zwischen den beiden Prothesenköpfen eine bessere Funktion und eine Verminderung des Abriebs im Acetabulum bewirken soll. Indikationen zur Implantation einer Duokopfprothese sind Schenkelhalsfrakturen beibiologisch alten $(>75$ bis 80 Jahre), deutlich vorerkrankten $\mathrm{Pa}$ tienten mit niedrigem Aktivitätsniveau. Vorteile der Duokopfprothese sind die kürzere Operationsdauer und der geringere Blutverlust durch die fehlende Pfannenpräparation. Außerdem ist die Ope- 
rationstechnik ohne Pfannenpräparation einfacher und auch durch weniger erfahrene Operateure sicher durchführbar. In einer Studie von Kannan et al. [20] konnte gezeigt werden, dass die Reoperationsrate im längerfristigen Verlauf nach Implantation von bipolaren Prothesen signifikant niedriger war als bei TEP. Ein weiterer Vorteil der Duokopfprothese ist das niedrigere Luxationsrisiko durch den größeren Kopfdurchmesser im Vergleich zur TEP [18]. Daher sollte bei Patienten mit unsicherer Compliance im Hinblick auf die postoperative Luxationsprophylaxe (z. B. bei Demenz) eine Duokopfprothese implantiert werden.

Allerdings ist die Funktion nach Implantation einer Duokopfprothese meist schlechter als bei der TEP [18]. Bei aktiven Patienten mit stärkerer Beanspruchung der Prothese kann des Weiteren als seltene Komplikation im Verlauf eine Protrusion des Prothesenkopfs durch das Acetabulum ins kleine Becken auftreten. Aus diesen Gründen ist bei älteren, jedoch noch mobilen Patienten mit hohem funktionellem Anspruch die Indikation zur Implantation einer TEP gegeben. Auch eine bereits vor der Fraktur bestehende symptomatische Koxarthrose auf der betroffenen Seite stellt eine Indikation zur TEP dar.

\section{Zementierte versus unzementierte Implantation}

Die endoprothetische Versorgung von Schenkelhalsfrakturen kann sowohl zementiert als auch unzementiert erfolgen.

Eine potenzielle Gefahr bei der zementierten Technik ist das sog. BoneCement-Implantation-Syndrom (BCIS). Dabei handelt es sich um eine Zementreaktion, die beim Einbringen des Zements oder dem anschließenden Einsetzen der Prothese auftritt und sich durch Symptome wie Hypoxie, Hypotension, Herzrhythmusstörungen, erhöhten pulmonalen Gefäßwiderstand bis hin zum Herzstillstand äußert $[9,28]$. Es werden je nach Ausprägung 3 Schweregrade des BCIS unterschieden [9]. In den unterschiedlichen Ausprägungen sind bis zu $28 \%$ aller Patienten mit Implantation zementierter Duokopfprothesen vom BCIS betroffen, wobei schwere Formen des BCIS mit einer deutlich erhöhten Mortalität einher-

Trauma Berufskrankh 2017 · 19 (Suppl 2):S192-S197 DOI 10.1007/s10039-017-0246-X

(c) Springer Medizin Verlag Berlin 2017

J. Hack · B. Bücking · D. Eschbach · S. Ruchholtz

Hüftendoprothetik nach Trauma. Was sind die „Big Points“?

\section{Zusammenfassung}

Hintergrund. Schenkelhalsfrakturen sind typische Frakturen des älteren Menschen, deren Häufigkeit aufgrund des zunehmenden Anteils älterer Menschen an der Bevölkerung in Zukunft weiter steigen wird. Sowohl bei der operativen Behandlung als auch beim perioperativen Management sind aufgrund des größtenteils geriatrischen Patientenguts einige Besonderheiten zu beachten.

Ziel der Arbeit. Im vorliegenden Beitrag sollen die verschiedenen operativen Therapieoptionen sowie die Besonderheiten in der perioperativen Betreuung von Patienten mit Schenkelhalsfraktur dargestellt werden. Material und Methoden. Anhand einer selektiven Literaturrecherche werden die aktuell im klinischen Alltag etablierten Vorgehensweisen dargestellt und diskutiert. Ergebnisse und Diskussion. Die operative Therapie bei Schenkelhalsfraktur sollte so schnell wie möglich (innerhalb von $24 \mathrm{~h}$ nach dem Unfall) erfolgen. Bei dislozierten Frakturen (Garden III und IV) geriatrischer Patienten wird die Implantation einer zementierten Prothese empfohlen. Bei jüngeren und noch aktiven Patienten wird aufgrund der besseren funktionellen Ergebnisse die Totalendoprothese bevorzugt, während bei älteren, multimorbiden Patienten mit bereits vor der Fraktur eingeschränkter Mobilität in den meisten Fällen eine zementierte Duokopfprothese implantiert wird. Eine geriatrische Mitbetreuung während des stationären Aufenthalts und eine anschließende geriatrische Reha-Maßnahme verbessern das Outcome der Patienten.

\section{Schlüsselwörter}

Schenkelhalsfraktur · Proximale Femurfraktur . Alterstraumatologie - Geriatrische Fraktur . Gelenkersatz

\section{Hip arthroplasty after trauma. What are the big points?}

\section{Abstract}

Background. Femoral neck fractures are typical fractures of the elderly and the frequency is even expected to increase in the future because of the aging society. Due to the high number of comorbidities, there are special challenges concerning the operative treatment as well as the perioperative management of the mostly geriatric patients. Objective. The purpose of this article is to illustrate the different operative treatment options and the special requirements concerning the perioperative management of geriatric patients with femoral neck fractures. Material and methods. Based on a selective literature search, the procedures currently established in clinical practice are presented and discussed.

Results and conclusion. Surgical treatment should be performed as soon as possible (within $24 \mathrm{~h}$ ) after the trauma. In displaced fractures (Garden types III and IV) in geriatric patients implantation of a cemented prosthesis is recommended. Total endoprostheses are used in younger and active patients because of better functional results, whereas older multimorbid patients with limited mobility even before the fracture, benefit from dual head prostheses. Multidisciplinary orthogeriatric treatment during the acute inpatient treatment and orthogeriatric rehabilitation programs improve the outcome of patients.

\section{Keywords}

Femoral neck fracture $\cdot$ Hip fracture $\cdot$ Geriatric trauma - Geriatric fracture - Joint replacement gehen [28]. Als Ursache für das BCIS werden aktuell mehrere Theorien diskutiert, am ehesten scheinen zementassoziierte Embolien ursächlich, die einerseits mechanische Komplikationen verursachen und andererseits durch Mediatoraktivierung mit Freisetzung von vasoaktiven und proinflammatorischen Substanzen den pulmonalen Gefäßwiderstand erhöhen [9].

Neben diesen potenziellen Gefahren durch die Zementapplikation muss jedoch betont werden, dass die zementierte Technik das Risiko für periprothetische Frakturen während der Operation und im Verlauf deutlich reduziert. In einer Studie von Langslet et al. [25] trat bei 


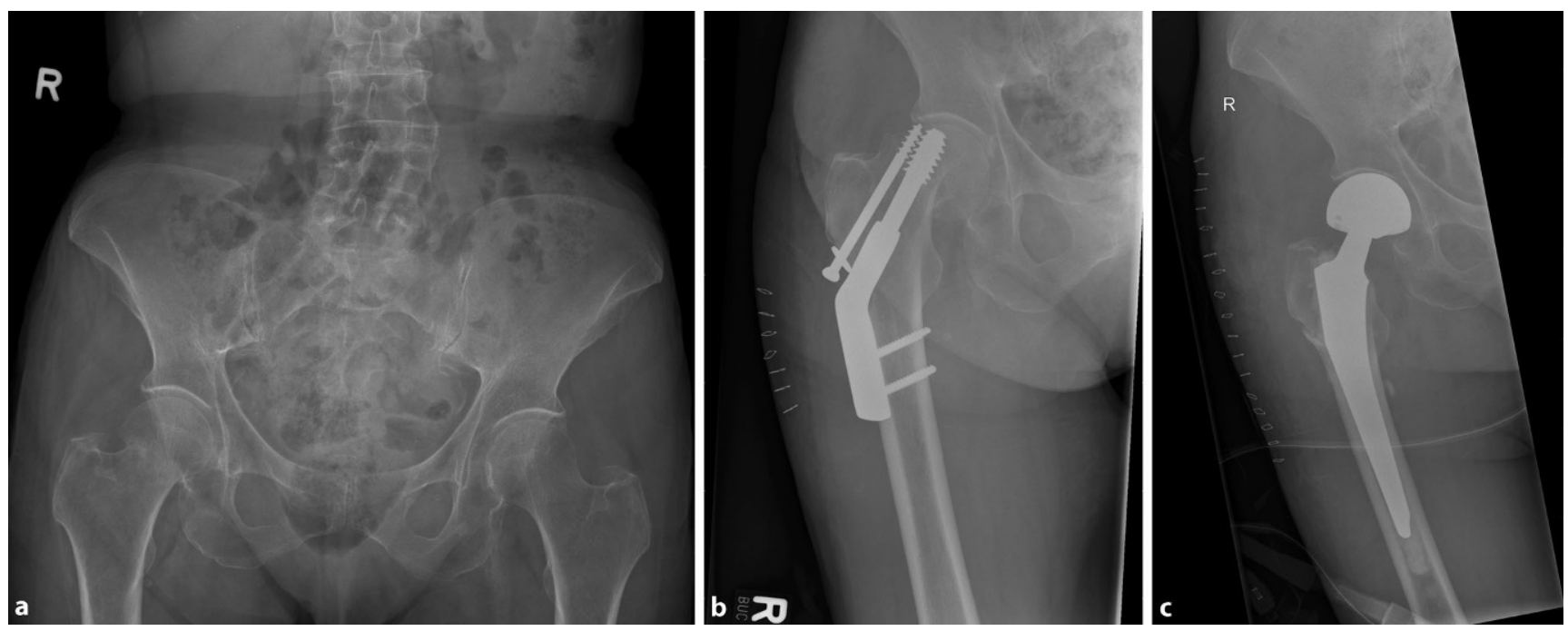

Abb. 1 ॥ Osteosyntheseversagen bei mit dynamischer Hüftschraube (DHS) versorgter Schenkelhalsfraktur Typ Garden I. a Präoperativ. b Osteosyntheseversagen nach Versorgung mit DHS. c Wechsel auf Duokopfprothese

7,9\% der unzementierten Duokopfprothesen verglichen mit $0,9 \%$ der zementierten Duokopfprothesen innerhalb von 5 Jahren nach Implantation eine periprothetische Fraktur auf. In einer Studie aus dem norwegischen Endoprothesenregister zeigte sich eine deutlich bessere 5-Jahres-Überlebensrate für zementierte als für zementfreie Duokopfprothesen ( $97 \%$ vs. $91 \%$ ), wobei periprothetische Frakturen und aseptische Lockerungen die häufigste Revisionsursache bei unzementierten Prothesen darstellten [16].

Aufgrund des deutlich erhöhten Risikos für periprothetische Frakturen bei unzementierten Prothesen sollte die Implantation des Prothesenschafts bei Schenkelhalsfrakturen also standardmäßig in der zementierten Technik erfolgen. Dabei sollten jedoch Maßnahmen zur Verringerung des Risikos eines BCIS ergriffen werden, z. B. gepulste Markraumlavage, Einbringen eines Einlüftungsschlauchs vor Zementapplikation, retrogrades Einbringen des Zements über eine Zementpistole und vorherige Kommunikation mit dem Anästhesisten [33].

Unter Beachtung dieser Maßnahmen ist die Krankenhausmortalität bei zementierter Hüftprothese laut einer Studie von Costa et al. [7] nicht erhöht im Vergleich zur unzementierten Hüftprothese.

\section{Nachbehandlung}

Postoperativ ist beim geriatrischen Schenkelhalsfrakturpatienten eine intensive physiotherapeutische Betreuung mit möglichst frühzeitiger Mobilisierung zur Vermeidung von immobilitätsassoziierten Komplikationen entscheidend. Da eine Teilbelastung bei geriatrischen Patienten in den meisten Fällen nicht praktikabel ist und oft zur kompletten Immobilisierung führt, sollte die operative Versorgung eine schmerzadaptierte Vollbelastung erlauben.

\section{Perioperatives Management}

\section{Zeitfaktor}

Eine besondere Herausforderung bei der Behandlung von Schenkelhalsfrakturen stellt das perioperative Management der meist geriatrischen Patienten mit deutlich erhöhtem Operationsrisiko dar.

Aufgrund der häufig vorliegenden Multimorbidität konkurrieren optimale Einstellung der Grunderkrankungen und Zeitfaktor miteinander. Einerseits sollte präoperativ zur Senkung des Narkoserisikos eine möglichst optimale Einstellung der Grunderkrankungen erfolgen, andererseits sollte die operative Therapie so schnell wie möglich erfolgen, um immobilitätsassoziierte Komplikationen wie Pneumonie, Thrombosen/Embolien und Dekubitalulzera zu vermeiden.
Da es deutliche Hinweis gibt, dass intra- und postoperative Mortalität und Morbidität relevant ansteigen, wenn die operative Therapie später als $24 \mathrm{~h}$ nach dem Unfall erfolgt $[8,23,35,36]$, sollte die Abklärung von Vorerkrankungen die operative Therapie nicht verzögern. Allgemeine, kurzfristig durchführbare Maßnahmen wie Ausgleich von Elektrolytstörungen, Hyper- oder Hypoglykämien, Verbesserung der Kreislaufsituation z. B. durch Volumengabe bei Exsikkose oder Bluttransfusionen bei relevanter Anämie sind jedoch unbedingt indiziert und laut aktueller AWMF (Arbeitsgemeinschaft der Wissenschaftlichen Medizinischen Fachgesellschaften)-Leitlinie empfohlen [1]. Eine kardiologische Abklärung hingegen ist nur in Einzelfällen bei Vorliegen von kardialen Vorerkrankungen indiziert, sofern dadurch die operative Therapie nicht unnötig verzögert wird, und wird nicht routinemäßig empfohlen [1].

Zusammenfassend sollte die operative Versorgung von Schenkelhalsfrakturen innerhalb von $24 \mathrm{~h}$ nach dem Unfall erfolgen, da bei späterer Versorgung das Mortalitäts- und Morbiditätsrisiko deutlich ansteigt. Wenn dies in speziellen Situationen (z. B. Einnahme von direkten oralen Antikoagulanzien, s. unten) nicht möglich ist, sollte die Operation spätestens innerhalb von $48 \mathrm{~h}$ erfolgen. 


\section{Gerinnungsmanagement}

Bei der Operationsvorbereitung ist ein besonderes Augenmerk auf eine evtl. vorliegende Antikoagulation zu richten. Bei den verschiedenen Wirkstoffgruppen sind unterschiedliche Vorgehensweisen indiziert.

Thrombozytenaggregationshemmer wie ASS und Plavix ${ }^{\circledR}$ werden perioperativ nicht abgesetzt, da einerseits die Blutungsneigung durch Absetzen kurz vor der Operation aufgrund der Lebensdauer der Thrombozyten nicht relevant verringert werden kann und das Blutungsrisiko bei alleiniger Einnahme von Plättchenaggregationshemmern in der Regel überschaubar ist und andererseits die möglichen thromboembolischen Komplikationen durch präoperatives Absetzten schwerwiegend sein können [10].

Bei Cumarinderivaten kann der INR (International Normalized Ratio)-Wert präoperativ mit Vitamin-K-Präparaten und notfalls Prothrombinkonzentrat (PPSB) erfolgreich angehoben und perioperativ ein Bridging mit HeparinPräparaten durchgeführt werden [4].

In den letzten Jahren finden direkte orale Antikoagulanzien (DOACs) vermehrt Anwendung. Da diese Präparate relativ neu sind, liegen erst wenige Daten zum Vorgehen bei Notfalloperationen vor. Problematisch ist die Tatsache, dass für DOACs bisher nur ein (sehr teurer) spezifischer Antagonist für Dabigatran (Idarucizumab) verfügbar ist. Laut aktueller Literatur sollte die letzte Einnahme von DOACs mindestens $12 \mathrm{~h}$, idealerweise jedoch $24 \mathrm{~h}$ zurückliegen [24, 37]. Postoperativ sollte die Einnahme nach $24-48 \mathrm{~h}$ wieder begonnen werden, sofern bis dahin eine adäquate Hämostase stattgefunden hat; ein Bridging ist nicht erforderlich [24].

Bei Einnahme von Dabigatran $\left(\right.$ Pradaxa $^{\circledR}$ ) ist die Nierenfunktion besonders zu berücksichtigen. Dabigatran wird hauptsächlich renal eliminiert, sodass auf eine ausreichende Diurese geachtet werden muss. Bei eingeschränkter Nierenfunktion ist die Halbwertszeit dementsprechend verlängert. Bei akuten Blutungskomplikationen muss Idarucizumab gegeben oder eine Dialyse erwogen werden [24]. Die Wirkung von Rivaroxaban $\left(\right.$ Xarelto $^{\circledR}$ ) konnte in einer Studie an gesunden Probanden im Gegensatz zu Dabigatran durch Gabe von PPSB sofort komplett antagonisiert werden [12]. Der Einsatz von PPSB wird aktuell jedoch nur bei Auftreten von schwerwiegenden Blutungskomplikationen und nicht routinemäßig empfohlen [24].

Rivaroxaban kann im Gegensatz zu Dabigatran nicht durch Dialyse eliminiert werden [24].

Aufgrund der fehlenden Erfahrungswerte mit DOACs ist im Zweifel die Rücksprache mit einem Gerinnungsphysiologen empfehlenswert.

\section{Geriatrische Mitbetreuung}

In der perioperativen Behandlung von geriatrischen Patienten mit Schenkelhalsfraktur hat sich eine geriatrische Mitbetreuung bewährt. Hierbei sind verschiedene Varianten möglich, von regelmäßigen gemeinsamen Visiten mit einem Geriater, einem konsiliarisch tätigen Geriater bis hin zu gemeinsamen Stationen [5, 32].

Auch spezielle geriatrische RehaMaßnahmen in direktem Anschluss an den akutstationären Aufenthalt haben einen positiven Einfluss auf das Outcome der Patienten [2].

\section{Outcome/Prognose}

Etwa ein Drittel der geriatrischen Patienten verstirbt innerhalb des ersten Jahres $[3,21]$, ein weiteres Drittel wird neu institutionalisiert [13], und 5-9\% der Patienten behalten bleibende funktionelle Einschränkungen zurück [26, 38]. Das Risiko, innerhalb eines Jahres eine weitere osteoporosebedingte Fraktur zu erleiden, beträgt ca. $30 \%$ [34].

\section{Fazit für die Praxis}

- Schenkelhalsfrakturen sind typische
osteoporoseassoziierte Frakturen
älterer Patienten.
- In den allermeisten Fällen ist die
operative Therapie indiziert.
- Bei undislozierten Frakturen (Gar-
den I und II) kann eine osteosyn-

thetische Versorgung erfolgen, üblicherweise mittels DHS.

- Bei dislozierten Frakturen (Garden III und IV) erfolgt die endoprothetische Versorgung.

- Aufgrund des erhöhten Langzeitrisikos für periprothetische Frakturen bei unzementierten Prothesen wird eine zementierte Implantation des Prothesenschafts empfohlen.

- Bei jüngeren, aktiven Patienten erfolgt aufgrund der besseren Funktion meist die Implantation einer Totalendoprothese.

- Bei älteren, multimorbiden Patienten erfolgt meist die Implantation einer Duokopfprothese.

- Die osteosynthetische Versorgung sollte innerhalb von $6 \mathrm{~h}$, die endoprothetische Versorgung innerhalb von $24 \mathrm{~h}$ erfolgen.

- Eine geriatrische Mitbehandlung während des akutstationären Aufenthalts sowie geriatrische RehaMaßnahmen verbessern das Outcome.

\section{Korrespondenzadresse}

\section{Dr. J. Hack}

Zentrum für Orthopädie und Unfallchirurgie, Universitätsklinikum Gießen und Marburg $\mathrm{GmbH}$, Standort Marburg Baldingerstr., 35043 Marburg, Deutschland hackj@med.uni-marburg.de

\section{Einhaltung ethischer Richtlinien}

Interessenkonflikt. J. Hack, B. Bücking, D. Eschbach und S. Ruchholtz geben an, dass kein Interessenkonflikt besteht.

Dieser Beitrag beinhaltet keine von den Autoren durchgeführten Studien an Menschen oder Tieren.

The supplement containing this article is not sponsored by industry.

\section{Literatur}

1. AWMF-Leitlinie (2015) Schenkelhalsfraktur des Erwachsenen. http://www.awmf.org/uploads/tx szleitlinien/012-001I_S2e_Schenkelhalsfraktur_ 2015-10_01.pdf.Zugegriffen: 12. Feb 2017

2. Bachmann S, Finger C, Huss A et al (2010) Inpatient rehabilitation specifically designed for geriatric patients: systematic review and meta-analysis of randomised controlled trials. BMJ 340:c1718 
3. Bliemel C, Sielski R, Doering B et al (2016) Pre-fracture quality of life predicts 1-year survival in elderly patients with hip fracture - development of a new scoring system. Osteoporos Int 27:1979-1987

4. Buecking B, Eschbach D, Bliemel C et al (2014) Effectiveness of vitamin $\mathrm{K}$ in anticoagulation reversal for hip fracture surgery - a prospective observational study. Thromb Res 133:42-47

5. Buecking B, Timmesfeld N, Riem S et al (2013) Early orthogeriatric treatment of trauma in the elderly: a systematic review and metaanalysis. Dtsch Ärztebl Int 110:255-262

6. Buord JM, Flecher X, Parratte S et al (2010) Garden I femoral neck fractures in patients 65 years old and older: Is conservative functional treatment a viable option? Orthop Traumatol Surg Res 96:228-234

7. Costa ML, Griffin XL, Pendleton N et al (2011) Does cementing the femoral component increase the risk of peri-operative mortality for patients having replacement surgery for a fracture of the neck of femur? Data from the National Hip Fracture Database. J Bone Joint Surg Br 93:1405-1410

8. Davis FM, Woolner T, Frampton C et al (1987) Prospective multi-centre trial of mortality following general or spinal anaesthesia for hip fracture surgery in the elderly. Br J Anaesth 59:1080-1088

9. Donaldson AJ, Thomson AE, Harper NJ, Kenny NW (2009) Bone cement implantation syndrome. Br J Anaesth 102:12-22

10. American College of Chest Physicians, Douketis JD, Spyropoulos AC, Spencer FA et al (2012) Perioperative management of antithrombotic therapy: antithrombotic therapy and prevention of thrombosis, 9th ed.: American College of Chest Physicians evidence-based clinical practice guidelines. Chest 141(2 Suppl):e326S-e350S

11. DVO (2014) DVO Leitlinie Osteoporose. http://www.dv-osteologie.org/uploads/ Leitlinie\%202014/DVO-Leitlinie\%200steoporose $\% 202014 \% 20$ Kurzfassung $\% 20$ und\%20Langfassung \%20Version\%201a\%2012\%2001\%202016.pdf. Zugegriffen: 12. Feb 2017

12. Eerenberg ES, Kamphuisen PW, Sijpkens MK et al (2011) Reversal of rivaroxaban and dabigatran by prothrombin complex concentrate: a randomized placebo-controlled, crossover study in healthy subjects. Circulation 124:1573-1579

13. Fisher AA, Davis MW, Rubenach SE et al (2006) Outcomes for older patients with hip fractures: the impact of orthopedic and geriatric medicine cocare. JOrthop Trauma 20:172-178

14. Garden RS (1964) Stability and union in subcapital fractures of the femur. J Bone Joint Surg $\mathrm{Br}$ 46:630-647

15. Gesundheitsberichterstattung des Bundes (2014) Diagnosedaten der Krankenhäuser ab 2000. https://www.gbe-bund.de/oowa921-install/ servlet/oowa/aw92/dboowasys921.xwdevkit/ xwd init?gbe.isgbetol/xs start neu/\&p aid $=3 \&$ p_aid $=23657809 \&$ nummer $=702 \&$ p_sprache $=D \&$ p_indsp=522\&p_aid=99215671\#SVG. Zugegriffen:25. Okt 2014

16. Gjertsen JE, Lie SA, Vinje T et al (2012) More re-operations after uncemented than cemented hemiarthroplasty used in the treatment of displaced fractures of the femoral neck: an observational study of 11,116 hemiarthroplasties from a national register. J Bone Joint Surg $\mathrm{Br}$ 94:1113-1119

17. Gjertsen JE, Vinje T, Engesaeter LB et al (2010) Internal screw fixation compared with bipolar hemiarthroplasty for treatment of displaced femoral neck fractures in elderly people. J Bone Joint Surg Am 92:619-628
18. Hopley C, Stengel D, Ekkernkamp A, Wich M (2010) Primary total hip arthroplasty versus hemiarthroplasty for displaced intracapsular hip fractures in older patients: systematic review. BM 340:c2332. doi:10.1136/bmj.c2332

19. Kanis JA, Odén A, McCloskey EV et al (2012) A systematic review of hip fracture incidence and probability of fracture worldwide. Osteoporos Int 23:2239-2256

20. Kannan A, Kancherla R, McMahon S et al (2012) Arthroplasty options in femoral-neck fracture: answers from the national registries. Int Orthop 36:1-8

21. Keene GS, Parker MJ, Pryor GA (1993) Mortality and morbidity after hip fractures. BMJ 307:1248-1250

22. Konnopka A, Jerusel N, König HH (2009) The health and economic consequences of osteopeniaand osteoporosis-attributable hip fractures in Germany: estimation for 2002 and projection until 2050. Osteoporos Int 20:1117-1129

23. Kostuj T, Smektala R, Schulze-Raestrup U, MüllerMai C (2013) Einfluss des Operationszeitpunkts und -verfahrens auf Mortalität und Frühkomplikationen der Schenkelhalsfraktur. Unfallchirurg 116:131-137

24. Lai A, Davidson N, Galloway SW, Thachil J (2014) Perioperative management of patients on new oral anticoagulants. Br JSurg 101:742-749

25. Langslet E, Frihagen F, Opland V et al (2014) Cemented versus uncemented hemiarthroplasty for displaced femoral neck fractures: 5-year followup of a randomized trial. Clin Orthop Relat Res 472:1291-1299

26. Magaziner J, Simonsick EM, Kashner TM et al (1990) Predictors of functional recovery one year following hospital discharge for hip fracture: a prospective study. J Gerontol 45:M101-M107

27. Manninger J, Kazar G, Fekete G et al (1989) Significance of urgent (within $6 \mathrm{~h}$ ) internal fixation in the management of fractures of the neck of the femur.Injury 10:101-105

28. Olsen F, Kotyra M, Houltz E, Ricksten SE (2014) Bone cement implantation syndrome in cemented hemiarthroplasty for femoral neck fracture: incidence, risk factors, and effect on outcome. $\mathrm{Br}$ J Anaesth 113:800-806

29. Parker MJ, Gurusamy KS (2006) Internal fixation versus arthroplasty for intracapsular proximal femoral fractures in adults. Cochrane Database Syst Rev. doi:10.1002/14651858.cd001708.pub2

30. Parker MJ, Khan RJK, Crawford J, Pryor GA (2002) Hemiarthroplasty versus internal fixation for displaced intracapsular hip fractures in the elderly. JBone Joint Surg Br 84:1150-1155

31. Pauwels F (1935) Der Schenkelhalsbruch: Ein mechanisches Problem. Enke, Stuttgart

32. Prestmo A, Hagen G, Sletvold O et al (2015) Comprehensive geriatric care for patients with hip fractures: a prospective, randomised, controlled trial. Lancet 385:1623-1633

33. Rutter PD, Panesar SS, Darzi A, Donaldson LJ (2014) What is the risk of death or severe harm due to bone cement implantation syndrome among patients undergoing hip hemiarthroplasty for fractured neck of femur? A patient safety surveillance study. BMJOpen 4:e004853

34. Siebert HR, Beck A (2005) Unfallchirurgie im Alter. Chirurg 76:139-150

35. Simunovic N, Devereaux PJ, Sprague $S$ et al (2010) Effect of early surgery after hip fracture on mortality and complications: systematic review and meta-analysis. CMAJ 182:1609-1616

36. Smektala R, Hahn S, Schräder P etal (2010) Mediale Schenkelhalsfraktur: Einfluss des Verzögerungs- zeitpunkts auf die Ergebnisqualität. Unfallchirurg 113:287-292

37. Sunkara T, Ofori E, Zarubin V et al (2016) Perioperative management of Direct Oral Anticoagulations (DOACs): a systematic review. Health Serv Insights 13(Suppl 1):25-36

38. Vergara I, Vrotsou K, Orive M et al (2014) Factors related to functional prognosis in elderly patients after accidental hip fractures: a prospective cohort study. BMC Geriatr 14:124 